\title{
The contextual nature of rhythmic interpretation
}

\author{
STEPHEN HANDEL and GREGORY R. LAWSON \\ University of Tennessee, Knoxville, Tennessee
}

\begin{abstract}
Five experiments investigated the rhythmic interpretation of polyrhythms that consisted of two or three conflicting pulse trains (e.g., three elements/repetition vs. four elements/repetition vs. five elements/repetition). The preferred rhythms were either meter rhythms, in which one pulse train served as the background controlling beat breaking the repetition (i.e., measure) into equal parts, or cross-rhythms, in which two or three pulse trains were integrated into a foreground melodic rhythm. The choice among various meter rhythms and various cross-rhythms was contextual, being based on the polyrhythm configuration, the timing between elements of each pulse train, the frequency of the elements of each pulse train, as well as the duration or intensity accentuation of the elements of each pulse train. In addition, there were consistent individual differences in strategies for rhythmic interpretation. The diversity of rhythmic interpretations occurs only with sufficiently dense stimuli, and both experimentation and theorizing must approach this level of complexity.
\end{abstract}

Rhythmic organization is an inherent part of all human activity. It is as important in hammering a nail or playing basketball as it is in music or in dancing. Rhythm energizes, structures, creates, and expresses temporal quality. Moreover, rhythmic organization exists at all levels of activity. What musicians, carpenters, and dancers have in common is an appreciation of rhythmic levels. Musical rhythm emerges from the rhythmic lines of each instrument or instrumental section. Each line might be simple or complex, and yet, in a very real sense, the rhythm cannot be found at any one of these levels. Moreover, the emergent rhythm is multifaceted. There is the meter or beat, the pace or tempo, the accent pattern, and finally the melodic rhythm (Radocy \& Boyle, 1979). These aspects are not independent; they support each other and allow each to emerge.

By concentrating on rhythmic levels or "strata" (Yeston, 1976), we can deal with thick polyphonic passages in which several instruments are playing at once, entering the piece at different times, each with a different rhythm. One rhythmic line may be temporally related and consonant with the other rhythmic lines, or one rhythmic line may be temporally crossed and dissonant with the others. In these cases, there may or may not be a melodic line per se, and grouping and accentuation would be based on the interaction of several dimensions: timing, texture,

This research was supported by National Institute of Neurological and Communicative Disorders and Stroke Grant NS 14842. The authors would like to thank the reviewers for their helpful comments. Requests for reprints should be sent to Stephen Handel, Department of Psychology, University of Tennessee, Knoxville, Tennessee 37996-0900. duration, timbre, and orchestration. We would argue that rhythm emerges from the interplay of the various musical levels. The simple rhythmic concepts of meter, beat, accent, and phrase are not found at any single level (or melodic line) but arise out of the interactions among levels. In the same manner, more complex rhythmic structures such as combination. rhythms, crossed rhythms, and syncopated rhythms arise from the interplay among levels.

To demonstrate the utility of a theory of rhythm based on interacting levels, we have studied the rhythmic interpretation of syncopated polyrhythms. Polyrhythms are defined as the simultaneous presentation of two or more conflicting pulse trains. Each pulse train consists of a series of isochronous, identical elements. For a $3 \times 4 \times 5$ polyrhythm, the tone representing the 3 -pulse train would occur three times per pattern repetition (i.e., per measure), while the tones representing the 4- and 5-pulse trains would occur four and five times per pattern repetition. There would be a common point at which the elements of each pulse train coincided. Polyrhythms are ambiguous in that the rhythmic structure formed by the pulse trains is dissonant, since each pulse train proceeds' at a different rate. If the elements of one pulse train are differentiated to serve as the controlling meter evenly dividing the measure, then the elements of the other pulse trains will fall off the beat. Alternatively, two or even all three pulse trains may combine to yield a nonmeter cross-rhythm in which the timing between adjacent elements varies. The interplay of the pulse trains creates the competing, dissonant, rhythmic structures.

In previous work, Handel and Oshinsky (1981) investigated the meter interpretation of polyrhythms 
composed of two pulse trains. The results of this work illustrated that the meter or beat of a polyrhythm is dependent on perceptual interactions among the pulse trains and not on simple acoustical, temporal, or perceptual factors. There were qualitative differences in the meter interpretations for various polyrhythms. For some polyrhythms, meter preferences were based on the temporal interval between elements; for others, meter preferences were based on the interaction of timing and frequency. In all cases, however, meter varied as a function of the tempo. The rhythmic role of each pulse train, therefore, was a function of the context created by the two pulse trains.

Meter or beat interpretation represents but one kind of rhythmic interpretation. The beat divides duration into equal units and can be considered the regularizing component of rhythm. In contrast, melodic rhythms are infinitely variable, using the meter as background or pivot. In fact, the meter or beat and the melodic rhythm can be conceived as being intertwined, yet serving opposite functions.

To study rhythm, then, it is necessary to study all possible types of interpretation. It is for this reason that the purpose of the present experiment was to study rhythmic interpretation in general and not to restrict subjects' responses in any way. Subjects listened to a polyrhythm, and then tapped in synchrony to the perceived rhythm. In contrast to previous work, subjects were free to tap in synchrony to every element of the two or three pulse trains in a polyrhythm, creating a cross-rhythm. Alternatively, - as in previous work, subjects were free to tap in synchrony to some or all the elements of one pulse train, creating a meter rhythm.

This response freedom should allow a fuller view of the possibilities for rhythmic interpretation. Nonetheless, a tapping or shadowing response emphasizes the structural aspects of musical rhythm at the expense of the equally important experienced motional or emotional character of rhythms. Moreover, the synchronous response does not measure the intensity of the taps to particular elements. In spite of these shortcomings, tapping along with the rhythm is so transparent that musically naive as well as musically sophisticated subjects find it easy; it is as commonplace as listening. More significantly, the task allows subjects to respond to any rhythmic structure they perceive and prefer.

Five experiments are reported. Polyrhythms were composed of two- or three-pulse trains. In some cases, the elements were of equal intensity and duration. In other cases, the intensity and duration of the elements were varied to assess their effects on rhythmic interpretation. The results further confirmed that rhythm is emergent, arising from the context created by the interplay of the pulse trains. This context was determined by the timing among elements of each pulse train, the pitch, duration, and intensity of the elements, the configuration of the polyrhythm, and the overall tempo. In some instances, the rhythm was the complex cross-rhythm created by two or more pulse trains; in others, the rhythm was the meter created by one pulse train.

\section{GENERAL METHODOLOGY AND DATA ANALYSIS}

Several aspects of the methodology were common to all five experiments. These are described below. Details specific to each experiment will be described as needed.

\section{Methodology}

Subjects. All subjects were undergraduates at the University of Tennessee, recruited from introductory psychology classes. There was a wide range of musical training and ability. There were no differences in the rhythmic interpretation as a function of musical training or experience.

Polyrhythms. Across all experiments, there were five different polyrhythms composed of two pulse trains $-2 \times 3,2 \times 5,3 \times 4$, $3 \times 5$, and $4 \times 5-$ and three different polyrhythms composed of three pulse trains $-2 \times 3 \times 7,2 \times 5 \times 7$, and $3 \times 4 \times 5$. The frequency, amplitude envelope, and duration of the elements of each pulse train were controlled by separate oscillators (Wavetek Model 134) and voltage-controlled amplifiers (BRS Module AC251). If the frequencies of the elements of two or more pulse trains were identical, a single oscillator was used.

With the exception of Experiment 4, within each pulse train the duration of an individual element was equal to one-half of the onset-to-onset interval. For example, if the time for a pattern repetition was $2 \mathrm{sec}$, then the onset-to-onset interval between adjacent elements of a 5-pulse train would be $400 \mathrm{msec}$ and the duration of each element would be $200 \mathrm{msec}$. The amplitude-time envelope of each note consisted of a $40-\mathrm{msec}$ linear amplitude attack with a linear amplitude decay for the balance of the duration. For the above example, the attack time would be $\mathbf{4 0} \mathrm{msec}$ and the decay time would be $160 \mathrm{msec}$. Phenomenally, the sounds are mellow, as opposed to the more biting tones achievable with shorter attack lines.

Pattern repetition rates. Different rates of presentation were used. In terms of seconds per pattern repetition or seconds per measure, the rates ranged from 3.0 to $0.6 \mathrm{sec}$. These rates were chosen to sample the domain exhaustively. At the slow end of the continuum ( $3.0 \mathrm{sec} /$ pattern repetition), elements could be so separated in time that a continuing rhythm might not emerge; at the fast end of the continuum ( $0.6 \mathrm{sec} /$ repetition), the elements could occur so quickly that they might be perceived as forming subjective groups.

Experimental task. Subjects were told to tap along with the perceived rhythm in any way they wished. On each trial, a polyrhythm was presented for $15 \mathrm{sec}$, during which subjects merely listened. After a 5 -sec pause, the polyrhythm was repeated and subjects tapped a telegraph key along with the pattern to indicate the rhythm. The telegraph key triggered another audio oscillator, which was not audible to the subject. The clicking of the telegraph key was the only feedback available to the subject. Each pulse train was presented on one track by a four-channel tape recorder (TEAC A3440), while a second two-channel tape-recorder (Pioneer 707) simultaneously recorded the polyrhythm on one track and the subject's responses (i.e., the tone bursts triggered by the telegraph key) on the second track.

Stimulus presentation. The experimental session took place in a small room $(3 \times 4 \mathrm{~m})$ lined with acoustical tile. Subjects were seated $2.5 \mathrm{~m}$ from two or three speakers, stacked vertically, each of which presented one pulse train. Patterns were presented at a comfortable listening level, approximately $55 \mathrm{~dB}$ SPL. All subjects 
were tested individually, and were given frequent breaks to minimize fatigue.

In all experiments, the order of presentation was counterbalanced across subjects. Similar conditions (e.g., identical polyrhythms, identical rates) were randomized and distributed throughout the presentation. Pulse trains were presented equally often in each of the speakers and, if elements of each pulse train differed in frequency, each frequency was balanced across speakers. There was no effect on interpretation due to speaker position.

\section{Data Analysis}

Preliminary analyses indicated that there were three main response classes.

(1) The first class were responses in synchrony with all elements of two or three simultaneous pulse trains. This creates an irregular nonmetric rhythm as the temporal interval between adjacent elements varies. These are termed cross-rhythms and are specified in terms of the pulse trains, for example, $3 \times 4 \times 5$ or $3 \times 5$ crossrhythm.

(2) The second class were responses in synchrony with the elements of one of the pulse trains, that is, tapping every element of the 2-, 3-, 4-, 5-, or 7-pulse train. These are termed meter rhythms. A meter rhythm splits a pattern repetition or measure into equal parts. Sometimes, instead of tapping every element, subjects tapped every second or every third element of one pulse train. The most common response was tapping every other element of the 4 pulse train, or twice per measure. Since these responses indicated the salience of a given pulse train, they were combined in the analyses with the appropriate pulse train meter (e.g., tapping every other element of the 4-pulse train was considered a 4-pulse-train meter).

To specify the possible meters, we will use the following terminology. Typically, we will specify the meter in terms of a pulse train, e.g., a 3-pulse-train meter. Alternatively, we will specify the meter in terms of two possible response categories. First, subjects may tap the meter of the faster, more frequent pulse train in a polyrhythm (the 5 in the $4 \times 5$ polyrhythm, the 4 in the $3 \times 4$ polyrhythm). We will term this category faster meter (or, alternatively, slower meter if subjects tap the meter of the less frequent pulse train). Second, subjects may tap the meter of the low-pitch pulse train. We will term this category low-pitch meter (or, alternatively, high-pitch meter if the subjects tap the meter of the high-pitch pulse train).

(3) The third class were responses only on the cooccurrence of the two or three pulses, or once per pattern repetition (once per measure). These were termed unit rhythms.

The coding of responses was normally unambiguous at the slower presentation rates (e.g., $3 \mathrm{sec} /$ repetition to $1 \mathrm{sec} / \mathrm{repeti-}$ tion). At faster rates, subjects at times could not synchronize their tapping. The polyrhythm and response were displayed visually by an oscilloscope, and both authors independently coded the response. If the experimenters disagreed as to the coding of the response, the response was coded as a nonresponse. With rare exceptions (e.g., the $4 \times 5$ polyrhythm at the $.6-\mathrm{sec} /$ repetition rate), the percentage of nonresponses was less than $25 \%$.

Because previous work (Handel \& Oshinsky, 1981; Oshinsky \& Handel, 1978) had demonstrated individual differences in rhythmic interpretation, every subject's response was coded on the basis of the above classification. Thus, for a $2 \times 3 \times 7$ polyrhythm, the possible rhythms would be : (1) $2 \times 3 \times 7$ cross-rhythm; (2) 2-pulsetrain meter; (3) 3-pulse-train meter; (4) 7-pulse-train meter; (5) $2 \times 3$ cross-thythm; (6) $2 \times 7$ cross-rhythm; (7) $3 \times 7$ crossrhythm; (8) unit; and (9) a catch-all nonresponse category. Then a separate subject $\times$ subject overlap correlation matrix was constructed for each polyrhythm, indicating the agreement in rhythm interpretation among each pair of subjects. The overlap measure counted the number of identical rhythmic responses across experimental conditions generated by frequency, accentuation, and presentation rate. Matrices then were partitioned by trial and error to determine if there were clusters of subjects displaying contrasting patterns of rhythmic interpretation. The final step of the analysis was to determine the basis of rhythmic interpretation for each subject cluster.

\section{EXPERIMENT 1 \\ The Rhythmic Interpretation of Polyrhythms Composed of Two Pulse Trains}

The purpose of Experiment 1 was to investigate the rhythmic interpretation of polyrhythms composed of two pulse trains. To achieve generality, simple (e.g., $2 \times 3$ ) as well as complex (e.g., $4 \times 5$ ) polyrhythms were constructed (see Handel \& Oshinsky, 1981), and a wide range of presentation rates were used. The results demonstrated that the perceived rhythm was a function of the frequency relationships, the presentation rate, and the polyrhythm configuration.

\section{Method}

Subjects. There were 30 subjects.

Polyrhythms. Five different polyrhythms were used: $2 \times 3,2 \times 5$, $3 \times 4,3 \times 5$, and $4 \times 5$. The tones of the two pulse trains were either identical in frequency $(507 \mathrm{~Hz})$ or separated by a musical fourth such that in half the instances one pulse was $440 \mathrm{~Hz}$ (musical A4) and the second pulse was $586 \mathrm{~Hz}$ (musical D5) and in half the instances the frequencies of the pulse trains were reversed.

Pattern repetition rates. Six rates of presentation were used: 3.0, $2.4,2.0,1.6,1.2$, and .6 sec.

Experimental design. The entire experiment was composed of 90 conditions: 5 polyrhythms $\times 6$ presentation rates $\times 3$ frequency conditions (pulse trains were either identical in frequency or were separated by a musical fourth). Each subject was presented all 90 polyrhythms in a single 2-h session.

\section{Results}

The results section is organized into two parts. The first part discusses general principles underlying the use of cross-rhythms and meter rhythms. These response classes accounted for $98 \%$ of responses. The second part discusses strategies used by groups of subjects to interpret specific polyrhythms.

Cross-rhythms. Cross-rhythms are irregular because both pulse trains in combination define rhythm. The timing between adjacent elements coming from the two pulse trains varies, producing the uneven, nonregular rhythm. Given the perceptual complexity, as well as motoric demands in producing such cross-rhythms, it could be expected that the use of cross-rhythms would be maximum at the slower presentation rates and for the simpler polyrhythms ( $2 \times 3$ as opposed to $4 \times 5$ ).

In general, this was the outcome. For example, the percentage of cross-rhythms for the simple $2 \times 3$ polyrhythm was $81 \%$ at the $3.0-\mathrm{sec} /$ repetition rate, monotonically diminished to $58 \%$ at the $1.2-\mathrm{sec} /$ repetition rate, and down to $5 \%$ at the $.6-\mathrm{sec} /$ repetition rate. For the complex $4 \times 5$ polyrhythm, the percentage of cross-rhythms was $52 \%$ at the $3.0-\mathrm{sec} /$ repetition rate and down to less than $2 \%$ at both the 1.2- and .6-sec/repetition rates. 
Meter rhythms. A meter (or beat) rhythm divides a measure (or repetition) into equal time intervals; one of the pulse trains defines the time signature and the second pulse train becomes superimposed on the metric pulse train. The most important factor determining which pulse train will become the meter is the timing between elements. As found previously (Handel \& Oshinsky, 1981), there are rates so slow that the elements of a pulse train appear unconnected and are unsuited to serve as the meter. In that work, the interelement interval of $600-800 \mathrm{msec}$ appeared to be an upper bound to a meter. In addition, there are rates so fast that the elements of a pulse train are not heard separately but are perceived as grouped with subjectively accented and unaccented elements. The pulse train cannot serve directly as the meter. This transition point occurred when the interval between successive elements was about $200 \mathrm{msec}$. The same results were found here. For this reason, the use of the faster pulse-train meter (the 5 in a $2 \times 5$ polyrhythm) was maximum at the slower presentation rates, and the use of the slower pulse-train meter was maximum at the faster presentation rates. However, it was the configuration and not merely the presentation rate which determined the rhythmic interpretation. For example, whether a 4-pulse-train meter was perceived as the rhythm of the polyrhythm was not determined by its own rate, but was determined by the rhythmic configuration of which it was a part. For the $4 \times 5$ polyrhythm, the 5-pulse-train meter was preferred at slower rates, and the 4-pulse-train meter was preferred at the faster rates, but for the $3 \times 4$ polyrhythm, the 4-pulse-train meter was preferred at the slower rates and the 3-pulse-train meter was preferred at the faster rates.

A second subsidiary factor that affects the choice of meter is the preference for low-pitch elements. In the majority of cases, the choice of meter based on the timing constraints would be unambiguous: the elements of only one of the two pulse trains would fall within the timing window. However, when the elements of both pulse trains satisfy the 200- to 800 msec interelement interval constraints discussed previously, subjects shift to a meter based on the alternate cue of pitch. In all instances, the low-pitch pulsetrain meter was preferred to the high-pitch pulsetrain meter.

Interpretation strategies. Cross-rhythms and meters form the set of possible interpretations. Of equal importance, however, are the consistent interpretation strategies that groups of individuals utilized to achieve a rhythmic interpretation for a given polyrhythm. The configuration of a polyrhythm can affect the interpretation in two ways. First, the complexity of a polyrhythm can affect the availability and preference for cross-rhythms. Second, the rate of the pulse trains composing the polyrhythm can affect the choice of meter. The four major strategies which accounted for over $75 \%$ of the responses will be listed and described below. Other strategies were found, but these others were more complex and idiosyncratic.

The four strategies along with the number of subjects $(\mathrm{N})$ who followed each strategy were: (1) crossrhythms at the slowest rates, faster meters at the intermediate rates, and slower meters at the fastest rate found for the $2 \times 5$ and $3 \times 5$ polyrhythms $(N=12$ and $N=13$, respectively); (2) faster meters at the slower rates and slower meters at the faster rates found for all polyrhythms; (3) cross-rhythms at the slowest rates, low-pitch meters at the intermediate rates, and slower meters at the fastest rates found for the $3 \times 4$ and $4 \times 5$ polyrhythms $(N=9$ and $N=11$, respectively); and (4) cross-rhythms at the slower rates and low-pitch meters at the fastest rates found for the $2 \times 3$ polyrhythms $(\mathrm{N}=19)$.

What is important to note is that while the configuration of each polyrhythm will bring about different rhythmic interpretations, there are general factors which are common to all interpretations. To illustrate what is specific to each polyrhythm and what is common to all polyrhythms, the first rhythmic strategy will be discussed in detail below. Following this, the other strategies will be mentioned in turn.

(1) One common response was to tap in synchrony with all elements at the slowest rates, tap in synchrony with every element of the faster pulse train at the intermediate rates, and tap in synchrony with every element of the slower pulse train at the fastest rates. Two examples of this strategy-for the $2 \times 5$ and $3 \times 5$ polyrhythms-are shown in Figure 1 . Frequency did not affect the perceived rhythm, so all frequency conditions were combined.

Two points should be made about this response strategy. The first is that the type of rhythmic interpretation undergoes two changes when a switch from a cross-rhythm to a meter rhythm occurs: the rhythm changes from being irregular and based on two pulse trains (a melodic rhythm) to being regular and based on one pulse train.

The second point is that the transition points among the rhythms depended on the polyrhythm configuration as well as on the presentation rate. Consider first the effect of configuration. For the $3 \times 5$ polyrhythm, as compared with the $2 \times 5$ polyrhythm, the percentage of cross-rhythms was much smaller and the transition to the meter rhythm based on the 5-pulse train occurred at a slower rate. The $3 \times 5$ polyrhythm is far more rhythmically complex than the $2 \times 5$ polyrhythm. For this reason, it is more difficult to track the $3 \times 5$ configuration, and therefore the percentage of cross-rhythms decreased and the percentage of 5-pulse-train meter rhythms increased as compared with the $2 \times 5$ polyrhythm. This difficulty is accentuated at faster rates, so that the 


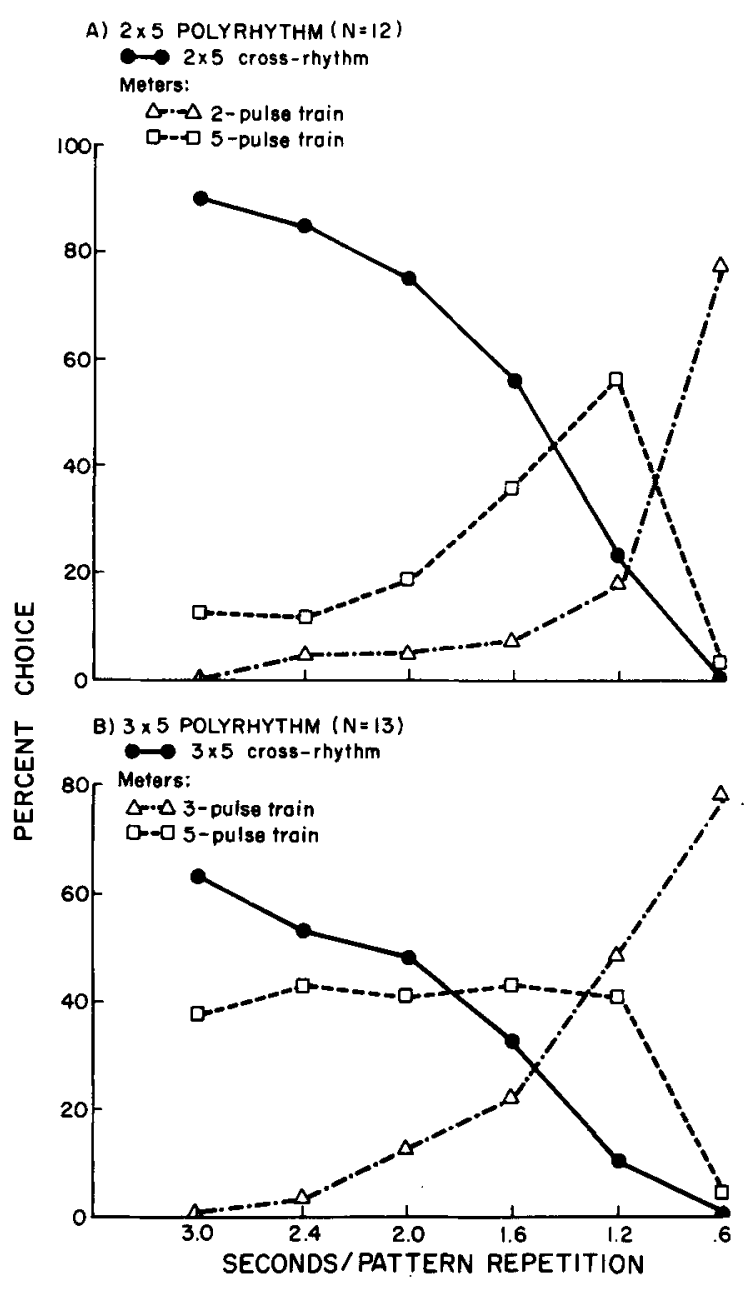

Figure 1. Rhythmic interpretations shifting from cross-rhythms to faster meter rhythms to slower meter rhythms.

transition to the 5-pulse train occurred at a slower rate. It is not simply that the $3 \times 5$ polyrhythm has more elements per repetition than the $2 \times 5$ polyrhythm (8 vs. 7). Rather, it is the complexity of the polyrhythm configuration which determines its usability as a rhythmic interpretation.

Consider now the effect of presentation rate. The timing between elements determines which pulse train will become the controlling meter. For the $3 \times 5$ polyrhythm, as compared with the $2 \times 5$ polyrhythm, the transition to the 3-pulse-train meter occurred at a slower rate than did the transition to the 2-pulse-train meter. As discussed previously, there are rates so slow that the elements of a pulse train appear unconnected and are therefore unsuited to serve as the meter; the interelement interval of $600-800 \mathrm{msec}$ appeared to be an upper bound to a meter. For these interpretations, the 3-pulse train was not chosen often as the meter rhythm until the presentation rate was below $1.6 \mathrm{sec} /$ repetition or an interelement interval of $533 \mathrm{msec}$, while the 2-pulse train was not chosen as the meter until the rate was below $1.2 \mathrm{sec} /$ repetition or an interval of $600 \mathrm{msec}$. In sum, the specifics of this rhythmic strategy were determined by the unique configuration of each polyrhythm as well as by general timing characteristics common to all polyrhythms and possibly to all temporal patterns.

The result that the unique configuration of each polyrhythm as well as general timing constraints jointly determine rhythmic interpretation was true for the other rhythmic strategies.

(2) For the second strategy, the transition between the meter based on the faster pulse train and the meter based on the slower pulse train was determined by timing constraints. The slower pulse-train meter was not chosen until the interelement interval was less than $600-800 \mathrm{msec}$, and the faster pulse-train meter was not chosen after the interelement interval dropped below 200 msec.

(3) For the third strategy, the transition from cross-rhythms, to low-pitch meters, to slower meters was based mainly on the timing constraints. For both the $3 \times 4$ and $4 \times 5$ polyrhythms, a cross-rhythm was much preferred at the slowest rates. Then, as the cross-rhythm became less preferred-at $1.6 \mathrm{sec} /$ repetition for the $4 \times 5$ polyrhythm and at $1.2 \mathrm{sec} /$ repetition for the $3 \times 4$ polyrhythm-a low-pitch meter became the preferred interpretation. (Note that the $4 \times 5$ polyrhythm is more complex, so the transition from a cross-rhythm to a meter rhythm occurred at a slower rate.) At the 1.6- and 1.2-sec/ repetition rates, at which pitch preferences were maximum, the elements of the 3-, 4-, and 5-pulse trains satisfy the $200-$ to $800-\mathrm{msec}$ interelement interval constraints discussed previously. The timing therefore does not provide a basis for meter choice, and subjects shift to the alternate cue of pitch. At the fastest rate $(.6 \mathrm{sec} /$ repetition), the elements of the faster pulse train tend to occur too quickly, so meter choice again was based on element timing. However, the polyrhythm configuration also determined the choice of low-pitch meters, since the two pulse trains of the polyrhythm must occur at similar rates $(3 \times 4$ or $4 \times 5)$ for low-pitch meters to arise. For polyrhythms in which the pulse trains move at more different rates (e.g., $3 \times 5$ ), low-pitch meters do not occur even when the timing constraints are satisfied for both pulse trains.

(4) The fourth strategy occurred mainly for the $2 \times 3$ polyrhythm and utilized a cross-rhythm at all but the fastest rate, at which point the preferred rhythm was a low-pitch meter. This strategy also can be understood in terms of the concepts detailed above: polyrhythm complexity and timing constraints. The $2 \times 3$ polyrhythm is the simplest one, so the subjects were able to utilize the cross-rhythm at all but the fastest rates. At the fastest rates, the elements of both the 2- and 3-pulse train satisfy the 
temporal constraints, so subjects shift to a meter determined by pitch.

Individual subject consistency. It is difficult to assess quantitatively the degree of individual consistency across all five polyrhythms. A small subset of the subjects did display consistent styles. For example, 8 of the 30 subjects consistently tapped crossrhythms at slow presentation rates and tapped meter rhythms at the faster rates. The rhythmic interpretation still depended on the polyrhythm, since these subjects preferred the faster or slower pulse train for the $2 \times 5$ and $3 \times 5$ polyrhythms but preferred the low-pitch pulse train for the $2 \times 3,3 \times 4$, and $4 \times 5$ polyrhythms. It appears that the configuration of the polyrhythm and the temporal constraints were the dominant characteristics in determining the perceived rhythm. Consistent individual strategies operated within those restrictions.

\section{Discussion}

The results clearly illustrate the contextual nature of rhythmic interpretation. The perceived rhythm was determined by the tempo, by the pitch of the elements, by polyrhythm configuration, and by individual strategies.

Two basic kinds of rhythms were found. The first was a cross-rhythm achieved by tapping in synchrony to every element of the polyrhythm. Both pulse trains compose the rhythm; it is a melodic rhythm. The availability of this rhythm depended on the complexity of the polyrhythm and the rate of presentation. More complex polyrhythms $(3 \times 5$ as opposed to $2 \times 3$ or $2 \times 5$ ) generate a more irregular rhythm that seems less available perceptually; the temporal pattern formed by the elements is too complex to serve as the rhythm. Increasing the rate of presentation accentuates this difficulty, the pattern occurring too quickly to grasp or tap motorically.

The second was a meter or beat rhythm achieved by tapping the elements of one pulse train. Here, one pulse train defined the rhythm with the other pulse train superimposed. The use of a meter rhythm was determined by the timing and frequency of the elements, as well as the availability of the competing cross-rhythm. For interelement intervals above 600$800 \mathrm{msec}$, the elements appeared disjointed and unconnected so that they could not serve as a meter. Conversely, for interelement intervals below 200$300 \mathrm{msec}$, the elements appeared to form subjective groups so that they could not serve as a meter. If both pulse trains satisfied these timing constraints, as happened with polyrhythms with similar pulse trains $(2 \times 3,3 \times 4,4 \times 5)$, subjects determined the meter on the basis of a low-pitch preference. The results found here are quite similar to those of Handel and Oshinsky (1981), who explicitly required a meter rhythm.

To summarize, tempo restricted the availability of cross-rhythms and meter rhythms. Element pitch contributed to meter preference when timing constraints were satisified. Polyrhythm configuration further restricted the availability of cross-rhythms and determined the use of timing and/or pitch meter rhythms. The use of pitch meters seems restricted to polyrhythms in which the pulse trains move at nearly the same rate $(2 \times 3,3 \times 4,4 \times 5)$, since there are presentation rates at which the timing constraints are met for other polyrhythms (e.g., the 2.4- to 1.2-sec/ repetition rates for the $3 \times 5$ polyrhythm), and yet pitch meters do not occur. Finally, individual strategies lead to preferences among the possible rhythmic interpretations.

Nonetheless, polyrhythms composed of two pulse trains are quite simple. They are not nearly as complex as African songs, which may have four or five contrasting pulse trains (see Jones, 1959). This simplicity must act to restrict the possible rhythmic interpretations and thereby limit our understanding of rhythm in general. It is for this reason that the second experiment utilized polyrhythms composed of three pulse trains. This allowed for greater variation in the configural, temporal, and frequency relationships among the pulse trains.

\section{EXPERIMENT 2 \\ The Rhythmic Interpretation of Polyrhythms Composed of Three Pulse Trains}

The purpose of Experiment 2 was to investigate the rhythmic interpretation of polyrhythms composed of three pulse trains. The results demonstrated that rhythmic interpretation differed for each polyrhythm, depending on the temporal interaction of the component pulse trains as well as the frequency relationships among the pulse trains.

\section{Method}

Subjects. There were 60 subjects.

Polyrhythms. Three different polyrhythms were used: $2 \times 3 \times 7$, $2 \times 5 \times 7$, and $3 \times 4 \times 5$. In one case, two pulse trains were "slow" in contrast to the remaining pulse train $(2 \times 3 \times 7)$; in the second case, two pulse trains were "fast" in contrast to the remaining pulse train (e.g., $2 \times 5 \times 7$ ); and in the third case, the three pulse trains were similar in rate $(3 \times 4 \times 5)$.

The tones of the pulse trains constructed a major triad: $252 \mathrm{~Hz}$ (C4), $330 \mathrm{~Hz}$ (E4), and $392 \mathrm{~Hz}$ (G4). There were a total of $13 \mathrm{fre}$ quency conditions. In six conditions, each pulse train had a different frequency. In six conditions, two pulse trains had the identical frequency and the third pulse train differed. In half of these cases, the two identical pulse trains were composed of $252 \cdot \mathrm{Hz}$ tones and the third pulse train was composed of $392-\mathrm{Hz}$ tones; in the three remaining cases, the frequencies were reversed. In the single remaining condition, all three pulse trains were identical in frequency $(330 \mathrm{~Hz})$.

Pattern repetition rates. Five rates of presentation were used: $3.0,2.4,2.0,1.6$, and $1.0 \mathrm{sec}$.

Experimental design. There were 65 conditions for each polyrhythm-13 frequency variations $\times 5$ presentation rates. Twenty subjects were presented all 65 conditions for a single polyrhythm in one 90 -min session. 


\section{Results}

As found for polyrhythms composed of two pulse trains, cross-rhythms and meter rhythms were the basic rhythmic interpretations. However, each polyrhythm brought about very different strategies of rhythmic interpretations and therefore will be discussed separately. The aim will be to illustrate the diversity of strategies as opposed to commonalities found for polyrhythms composed of two pulse trains.

Preliminary analyses indicated that, for each polyrhythm, the experimental conditions could be split into two complementary parts: (1) conditions in which all pulse trains differed in frequency or were identical in frequency-these first conditions illustrated the role of polyrhythm configuration on rhythmic interpretation; and (2) conditions in which two pulse trains were identical in frequency and one pulse train differed-these second conditions illustrated the role of pitch constrast on rhythmic interpretation.

$2 \times 3 \times 7$ polyrhythm. There were two strategies of interpretation for the $2 \times 3 \times 7$ polyrhythm.
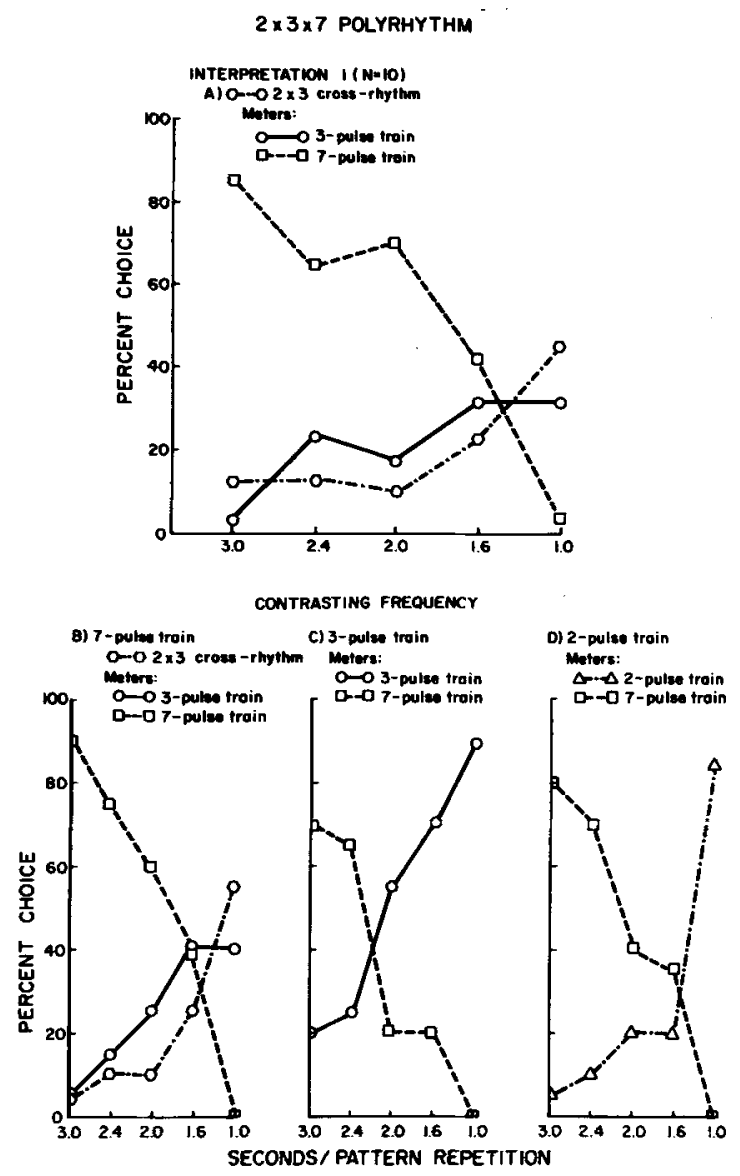

Figure 2. $2 \times 3 \times 7$ polyrhythm: Rythmic interpretation I based on 7-pulse-train meters, cross-rhythms, and slower pulse-train meters.
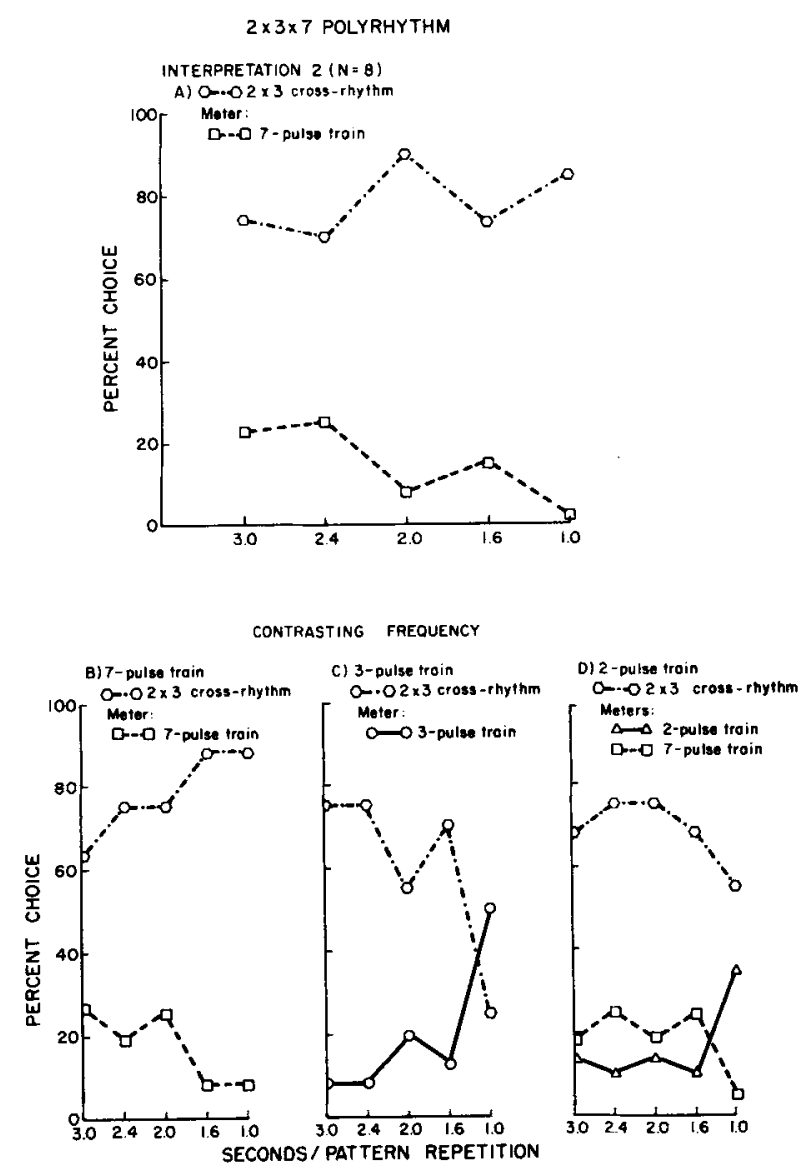

Figure $3.2 \times 3 \times 7$ polyrhythm: Rhythmic interpretation 2 based on $2 \times 3$ cross-rhythms.

(1) The first interpretation strategy is shown in Figure 2. For polyrhythms in which all pulse trains were identical or all differed in frequency (Panel A), a 7-pulse-train meter occurred at the slower rates and a $2 \times 3$ cross-rhythm or 3-pulse-train meter occurred at the faster rates. If two pulse trains were equal in frequency and one pulse train differed (Panels B, C, D), a meter based on the 7-pulse train was still preferred at the slower rates for all conditions. At the faster rates, the $2 \times 3$ cross-rhythm was still preferred if the 7-pulse train contrasted (Panel B). However, a meter rhythm based on the contrasting pulse train was preferred if the 2- or 3-pulse train differed (Panels C and D). In the latter two cases, pitch contrast between the 2- and 3-pulse trains combined with the pitch identity of the 2- and 7-pulse trains or of the 3- and 7-pulse trains acted to disallow the preferred $2 \times 3$ cross-rhythm. It is important to note that both conditions are necessary, since the $2 \times 3$ crossrhythm was preferred when all pulse trains differed in pitch.

(2) The second interpretation strategy is shown in Figure 3. These subjects preferred a rhythmic interpretation based on the $2 \times 3$ cross-rhythm. For all but 
one condition, the $2 \times 3$ cross-rhythm was preferred.

$2 \times 5 \times 7$ polyrhythm. There were two distinct response strategies for the $2 \times 5 \times 7$ polyrhythm. Each strategy was characterized by a meter preference.

(1) The first interpretation strategy was characterized by a preference for a 2-pulse-train meter, as shown in Figure 4. This preference was consistent across all presentation rates and conditions in which the elements of each polyrhythm differed in frequency or were identical in frequency (Panel A). In those cases in which two pulse trains were identical in frequency, a 2-pulse-train meter was preferred at faster rates. At the slower rates, if the 5- or 7-pulse train had the contrasting frequency (Panels $B$ and C), that pulse train gained in preference, roughly equaling the choice of the 2-pulse train. If the 2-pulse train had the contrasting pitch (Panel D), then the relatively weak rhythmic preference for the contrasting pulse-train meter reinforced the strong preference for a 2-pulse-train meter and that meter was invariably selected.

(2) The second interpretation strategy was characterized by a 7-pulse-train meter at the slower presentation rates and a 2-pulse-train meter rhythm at the faster rates. These outcomes are shown in Figure 5.

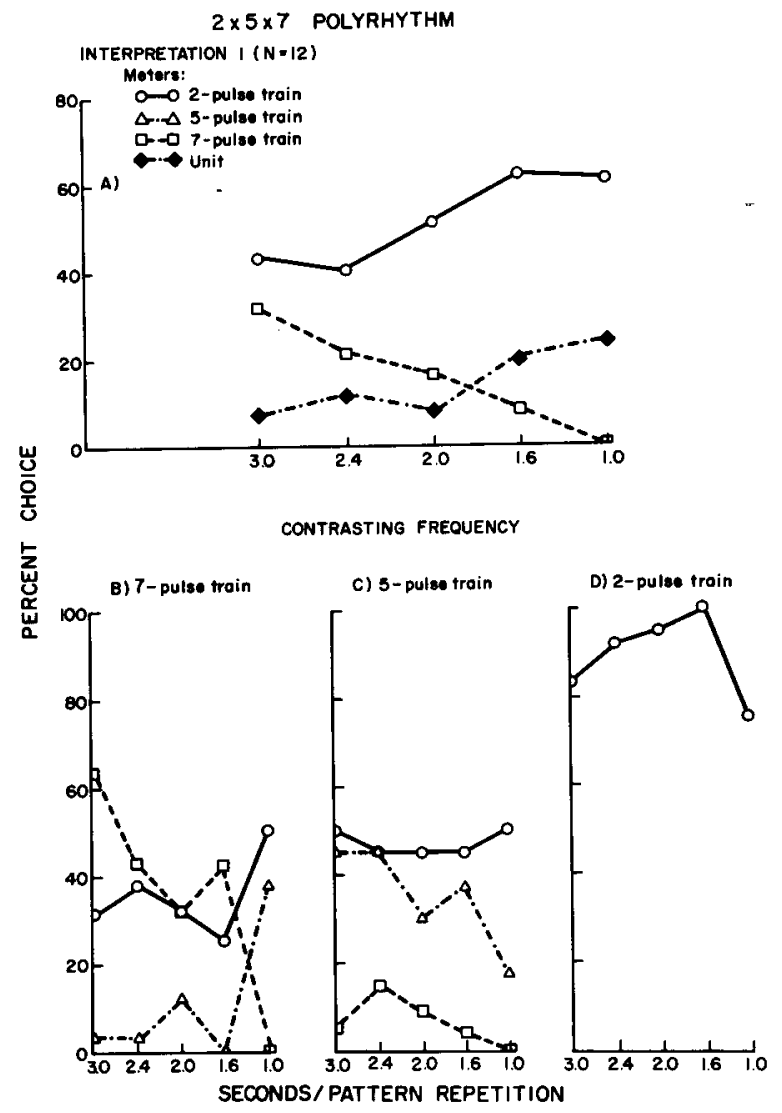

Figure 4. $2 \times 5 \times 7$ polyrhythm: Rhythmic interpretation I based on 2-pulse-train meter rhythms.
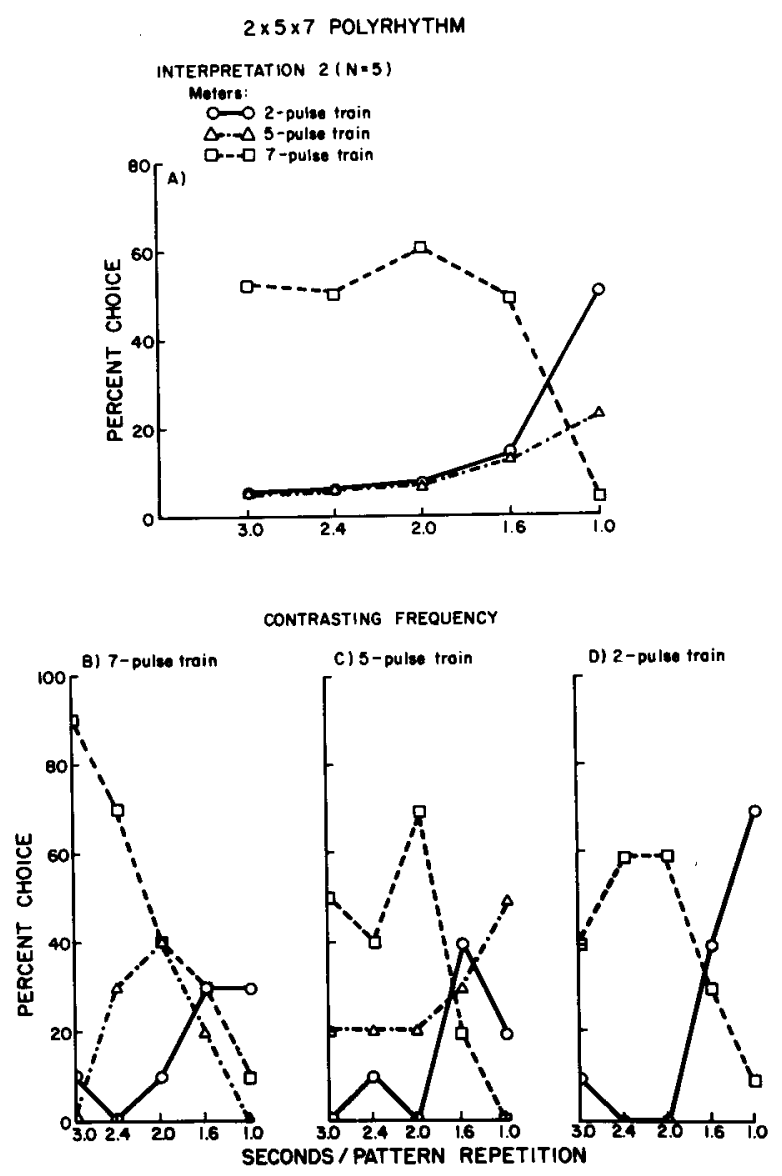

Figure 5. $2 \times 5 \times 7$ polyrhythm: Rhythmic interpretation 2 based on 7-and 2-pulse-train meter rhythms.

The only exception occurred when the 2- and 7-pulse trains had the same frequency and the 5-pulse-train meter differed (Panel C). In this case, although the 7pulse-train meter was still preferred at the slower rates, the 5-pulse-train meter was preferred at the faster rates.

$3 \times 4 \times 5$ polyrhythms. There were two very different response strategies found for the $3 \times 4 \times 5$ polyrhythms.

(1) The first interpretation strategy was characterized by a meter rhythm based on pitch, with the temporal characteristic of each pulse train of secondary importance, as shown in Figure 6. For these subjects, if all pulse trains had different frequencies, then the rhythmic interpretation was based on the meter created by the low-pitch element (Panel A). If two pulse trains had the same frequency, then the rhythmic interpretation was based on the meter created by the contrasting pitch, regardless of whether it was the high- or low-pitch element and regardless of pulsetrain rate (Panel B).

(2) The second interpretation strategy was characterized by cross-rhythms, as shown in Figure 7. If the elements of each pulse train had different frequencies 


\section{$3 \times 4 \times 5$ POLYRHYTHM}

INTERPRETATION I $(N=10)$

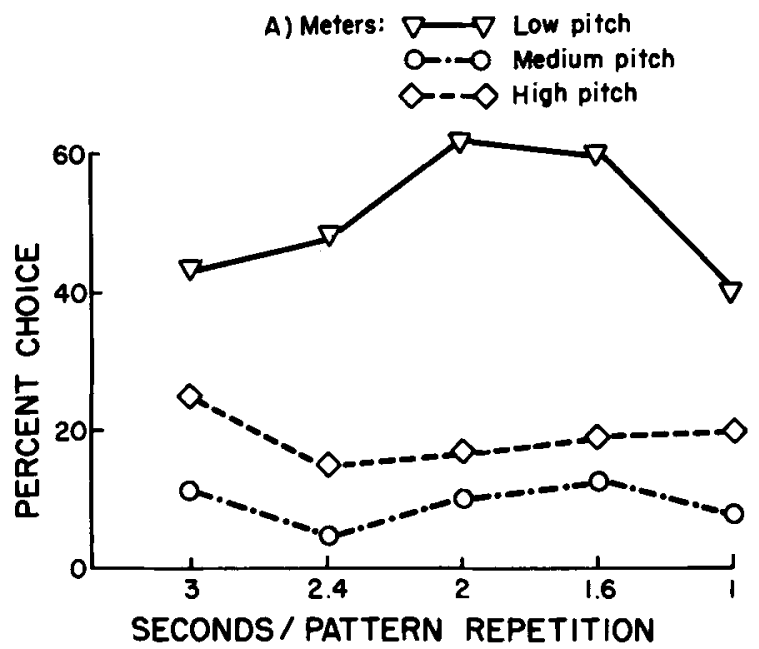

B) CONTRASTING FREQUENCY

Meters: $\Delta-\Delta$ Contrasting frequency 0 Identical frequencies

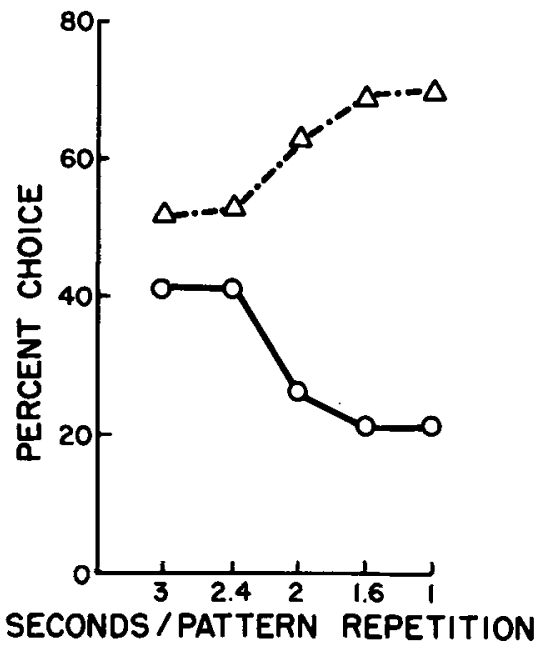

Figure 6. $3 \times 4 \times 5$ polyrhythm: Rhythmic interpretation I based on low-pitch and contrasting-frequency meter rhythms.

or the same frequency, then the $3 \times 4 \times 5$ crossrhythm was predominant down to a presentation rate of about $2.0 \mathrm{sec} / \mathrm{repetition,} \mathrm{at} \mathrm{which} \mathrm{time} \mathrm{a} \mathrm{meter}$ rhythm based on the 5-pulse train became preferred (Panel A). If the elements of two pulse trains had the same frequency, then subjects tapped in synchrony to the cross-rhythm created by the two identicalfrequency pulse trains at slower presentation rates, and tapped in synchrony with the meter created by the contrasting pulse train at the faster presentation rates.

\section{Discussion}

What does this all mean for a theory of rhythm? What it means generally is that any valid theory must be based on the properties of a specific rhythmic configuration. However, the rhythm is not invariant for any configuration; rather, it is simultaneously a function of tempo and frequency. Moreover, any theory must emphasize that rhythms exist at several levels and that listeners have access to many ways of interpreting a complex rhythm.

Specifically, the results using polyrhythms composed of three pulse trains illustrated that each polyrhythm will give rise to different unique patterns of rhythmic interpretation. At one extreme, the $3 \times 4 \times 5$ polyrhythm yielded meter rhythms based on element frequency, or yielded cross-rhythms composed of all three pulse trains or two identical-frequency pulse trains. At the other extreme, the $2 \times 5 \times 7$ polyrhythm yielded meter rhythms based on the slowest or on the fastest pulse train. The $2 \times 3 \times 7$ polyrhythm combined elements of both types of rhythmic strategies. One interpretation yielded cross-rhythms at all rates. In contrast, the second interpretation yielded meter rhythms based on the fastest pulse train at the slower rates and cross-rhythms plus slower pulse-train meters at the faster rates. These results further demonstrate that the rhythmic role of a single pulse train is a function of the configuration of the entire

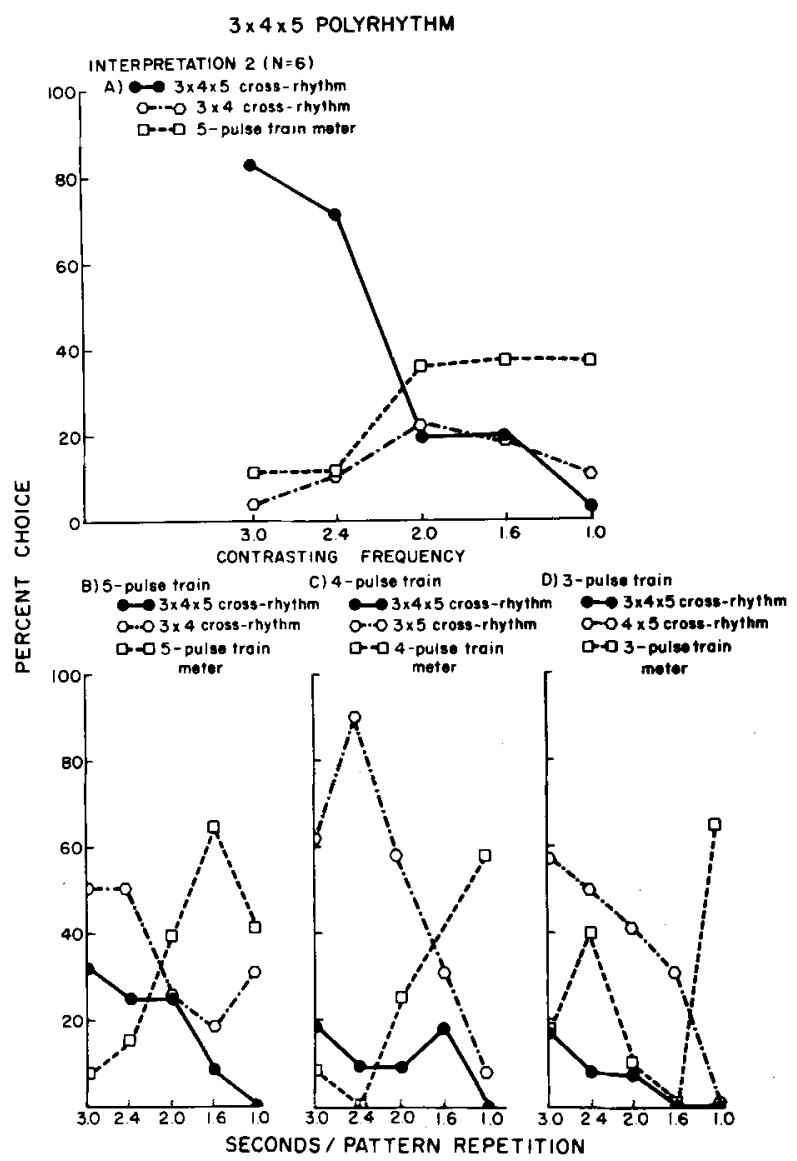

Figure $7.3 \times 4 \times 5$ polyrhythm: Rhythmic Interpretation 2 based on cross-rhythms and contrasting-frequency meter rhythms. 
polyrhythm and cannot be predicted from its own temporal characteristics.

Another way of illustrating the effect of the polyrhythm configuration as a whole is to compare the $2 \times 3$ polyrhythm with the $2 \times 3 \times 7$ polyrhythm and to compare the $2 \times 5$ polyrhythm with the $2 \times 5 \times 7$ polyrhythm. In each comparison, the 3-pulse-train polyrhythm is created by adding the 7-pulse train. What happened was that the 7-pulse train emphasized the rate similarity between the 2- and 3-pulse trains, thereby increasing the use of the $2 \times 3$ crossrhythm. However, this increased similarity precluded using either pulse train as a meter so that meter choice based on pitch preferences, as found for the $2 \times 3$ polyrhythm, did not occur. On the other hand, the 7-pulse train seemed to "strip" the 5-pulse train away from the 2 -pulse train: $2 \times 5$ cross-rhythms rarely occurred and 2-pulse-train meters occurred even at very slow presentation rates. The rhythmic consequences of the 7-pulse train depended on the configuration of the entire polyrhythm and dramatically changed the use of cross-rhythms or meters based on the other two pulse trains.

Nonetheless, there were similarities in the rhythmic interpretation among the polyrhythms. First, there were general timing factors common to all polyrhythms. Although there were exceptions, faster meters or cross-rhythms were preferred at slower rates, while slower meters were preferred at faster rates. Furthermore, the choice of meters was determined by the identical timing "window" found for polyrhythms composed of two pulse trains. Second, when two pulse trains had the same frequency and one differed, there was a transition to a meter based on the contrasting pulse train at the faster presentation rates. Third, there was a preference for a meter utilizing the low-pitch element. This tendency was most prominent for the $3 \times 4 \times 5$ polyrhythm, but the same preference existed for the other polyrhythms. These similarities reveal consistent timing factors operating within the constraints imposed by the polyrhythm configuration.

Differences in the kinds of rhythmic interpretations were not as obvious for rhythms composed of two pulse trains. For those polyrhythms, the rhythmic interpretations were more equivalent, although differences in the range of alternative rhythms and in the effect of element frequency did exist. It would appear that a certain level of complexity is required before configural effects become apparent. Otherwise, due to the simplicity of the stimulus itself, the possibilities for rhythmic interpretation are extremely limited.

The rhythm of these polyrhythms was generated by the interplay among equivalent pulse trains; individual elements were not accentuated. In other musical contexts, musical theorists have pointed out accentuation cues which appear to determine rhythm.
These would include intensity, duration, timbre, density, and melody (Cooper \& Meyer, 1960; Erikson, 1975; Yeston, 1976; Zuckerkandl, 1959). Accentuation cues exist at one level and act to modify the quality of that level. The purpose of the next three experiments was to utilize polyrhythms to investigate the role of duration and intensity accentuation in rhythmic interpretation. Elements within one or more pulse trains were to be accented and the resulting changes in rhythmic interpretation to be determined.

\section{EXPERIMENT 3 \\ The Effect of Element Duration on Rhythmic Interpretation}

The purpose of Experiment 3 was to investigate the effect of element duration on rhythmic interpretation of polyrhythms composed of two pulse trains. In the previous experiments, elements of each pulse train were of the same relative duration, one-half of the interval between the onsets of the adjacent elements. This is not necessarily true in music. Often the explicit rhythmic component is set off by some sort of duration accentuation. In the present experiment, a duration accent was produced by reducing all elements of one pulse train to $25 \mathrm{msec}$ in duration at all presentation rates. This duration was short enough to simulate a drum-tap or other impulsive percussive sound capable of carrying the meter or rhythm, yet long enough to give each element a distinctive tonal quality. The results found here, however, indicated that the effect of duration accentuation was not consistent, but changed as a function of the pitch relationships between the pulse trains.

\section{Method}

Subjects. There were 32 subjects.

Polyrhythms. Three polyrhythms were used: $2 \times 3,3 \times 4$, and $4 \times 5$. Tones of the two pulse trains were either identical in frequency $(507 \mathrm{~Hz}$ ) or separated by a musical fourth, such that in half the instances one pulse was $440 \mathrm{~Hz}$ (musical A4) and the second was $586 \mathrm{~Hz}$ (musical DS) and in half the instances the frequencies of the pulse trains were reversed.

The durations of the longer elements were equal to one-half the onset-to-onset interval (as in Experiments 1 and 2), and the durations of the shorter elements were equal to $25 \mathrm{msec}$. For the former elements, the amplitude-time envelope consisted of a 40 -msec linear amplitude attack with a linear amplitude decay for the balance of the duration. For the latter elements, the amplitude-time envelope consisted of a 12.5-msec linear amplitude attack and a 12.5msec linear amplitude decay.

Presentation rates. There were five presentation rates: $2.4,2.0$, $1.6,1.2$, and $.6 \mathrm{sec}$.

Experimental design. There were 10 frequency-duration conditions. In two conditions, one pulse train was composed of the long-duration elements and the second pulse train was composed of shorter duration elements, but all elements had the identical frequency. In four conditions, the frequencies of the pulse trains differed (i.e., one pulse train was low pitch, the other was high pitch), but the durations were identical (i.e., either both pulse trains had the longer duration elements or both pulse trains had the shorter duration elements). In the final four conditions, the frequency and duration of the elements of each pulse train differed (e.g., the ele- 
ments of one pulse train might have been longer duration and high pitch, so the elements of the second pulse train had to be shorter duration and low pitch).

The 10 frequency-duration conditions combined with the 5 presentation rates and 3 polyrhythms generated a total of 150 conditions. Each subject was presented all $\mathbf{1 5 0}$ conditions in a single 2h session.

\section{Results}

Preliminary analyses indicated that there were two basic response patterns. The first interpretation was found only for the $2 \times 3$ and $3 \times 4$ polyrhythms; subjects tapped every element of the polyrhythm (crossrhythm) at all but the fastest rate, and tapped the slower meter at the fastest rate $(\mathrm{N}=13$ for the $2 \times 3$ polyrhythm and $\mathrm{N}=7$ for the $3 \times 4$ polyrhythm). The second interpretation was found for all polyrhythms; subjects preferred the faster meter at the slower rates and the slower meter at the faster rates $\mathbf{N}=11$ for the $2 \times 3$ polyrhythm, $N=17$ for the $3 \times 4$ polyrhythm, and $N=22$ for the $4 \times 5$ polyrhythm). The results that follow will detail the second strategy, because it more clearly illustrated the effects of duration accentuation.

Consider first polyrhythms in which the elements of both pulse trains were of identical frequency. Here there was a preference for a meter rhythm utilizing the shorter element pulse train, overlaid on the basic preference for the faster meter at the slower presentation rates, and for the slower meter at the faster rates. This combination of effects is shown in Figure 8. The percentage choice of the slower meter was calculated by averaging the percentage choice of the 2-pulse train of the $2 \times 3$ polyrhythm, the 3-pulse train of the $3 \times 4$ polyrhythm, and the 4-pulse train of the $4 \times 5$ polyrhythm. The percentage choice of the faster meter was calculated in the same manner. At the slowest rate, there was a strong preference for the faster pulse train, but the effect of duration was minimal. At intermediate rates, there was a strong preference for a rhythm based on the shorter duration elements. The shorter element pulse train was chosen roughly twice as frequently as the longer duration pulse train. This outcome was true whether the shortduration pulse train was the faster pulse train $(65 \%$ to $32 \%$ ) or the slower pulse train $(61 \%$ to $30 \%)$. At the fastest rates, interelement timing again dominated the rhythmic iǹterpretation. Here, the slower meter was preferred and the percentage difference in meter choice between shorter and longer elements was reduced to $15 \%$.

Consider next polyrhythms in which the two pulse trains differed in frequency. Here, the rhythmic interpretation was based on a preference for the lowpitch meter and a timing preference for the faster meter at the slower rates and the slower meter at the faster rates. The effect of element duration was small and opposite in direction to that found when frequencies were identical; the longer duration element was slightly preferred.

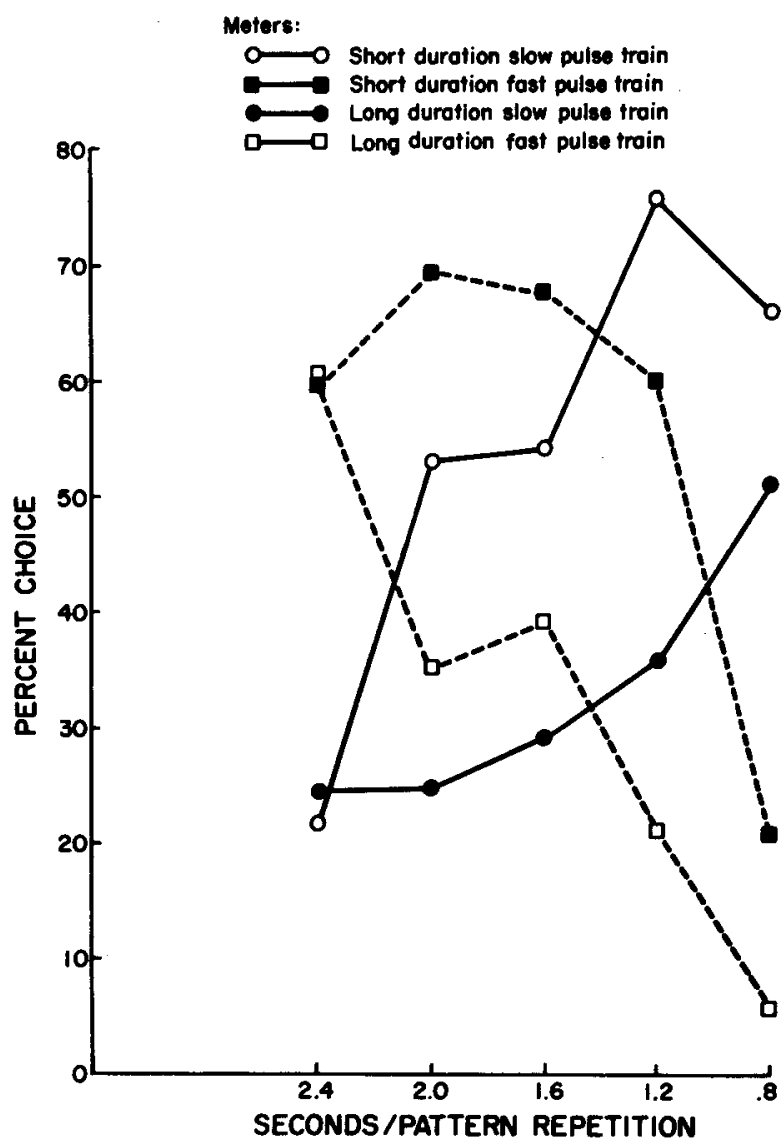

Figure 8. The effect of duration accentuation and pulse-train rate on meter choice when both pulse trains had the identical frequency.

The relationships among presentation rate, element frequency, and element duration are shown for the $3 \times 4$ polyrhythm in Figure 9 . In Panel A, the 3pulse train was the high-pitch tone and the 4-pulse train was the low-pitch tone. The open and closed circles give the percentage choice of the 3-pulse-train meter when elements of that pulse train were long and short. Similarly, the open and closed squares present the percentage choice of the 4-pulse-train meter when elements of that pulse train were long and short. In Panel B, the frequency relationships are reversed so that the 3-pulse train was low-pitch and the 4-pulse train was high-pitch. Again, the open and closed symbols represent long and short durations. It is clear that there was a preference for the low-pitch element, and that the use of the slower 3-pulse-train meter increased as the presentation rate was made faster. There was, however, little effect due to duration. Averaging over all conditions, the longer duration pulse-train meter was chosen $45 \%$ of the time and the shorter duration pulse-train meter was chosen $44 \%$ of the time. These results were typical for both the $2 \times 3$ and $4 \times 5$ polyrhythms. There was a strong preference for the low-pitch meter (54\% to 


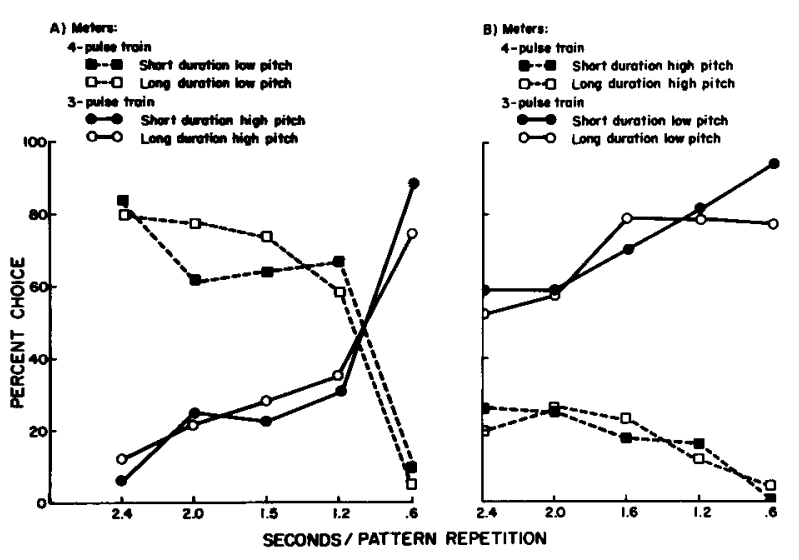

Figure 9. The effect of duration accentuation, element pitch, and pulse-train rate on meter choice.

$32 \%$ for the $2 \times 3$ polyrhythm and $63 \%$ to $27 \%$ for the $4 \times 5$ polyrhythm), and for both the polyrhythms the use of the slower pulse-train meter increased at faster presentation rates. There was little effect of duration. For the $2 \times 3$ polyrhythm, the percentage choice of long- and short-pulse-train meters was $48 \%$ and $42 \%$, respectively; for the $4 \times 5$ polyrhythm, the percentage choice of long- and short-pulse-train meters was $47 \%$ and $43 \%$, respectively. In sum, a meter based on the longer duration elements was just slightly preferred to a meter based on the shorter duration elements.

Individual subject consistency. There was a fair degree of individual consistency. For both strategies, there was a core of subjects who produced the identical response patterns across all three polyrhythms. For example, all 7 subjects who produced a crossrhythm (first interpretation) for the $3 \times 4$ polyrhythm also produced a cross-rhythm for the $2 \times 3$ rhythm. Similarly, 9 of the 11 subjects who tapped meter rhythms (second interpretation) for the $2 \times 3$ polyrhythm also tapped meter rhythms for both the $3 \times 4$ polyrhythm (of 17 subjects) and the $4 \times 5$ polyrhythm (of 22 subjects).

\section{Discussion}

The effect of element duration on rhythmic interpretation was inconsistent. If the two pulse trains were identical in frequency, then duration influenced rhythmic interpretation. The short, staccato elements tended to be perceived as the meter of the polyrhythm. This preference acted within the timing constraints imposed by the repetition rate. At the slowest and fastest rates, the perceived meter rhythm was constrained by the interelement interval. Only at the intermediate rates did duration affect rhythmic interpretation. In contrast, if the two pulse trains differed in frequency, then pitch preferences and timing constraints predominated and duration had little effect on rhythmic interpretation. There was a very slight preference for the longer duration elements, although this preference was very inconsistent. Duration became important in rhythmic interpretation only in the absence of other factors.

Perhaps the inconsistencies found here are indicative of the many possible ways that duration can function in music. Short elements can be used to indicate beats, or they can be merely "filler" notes between more important, longer accentuated notes. The role of other accentuation cues may be more clear-cut. For example, intensity accents are used musically to indicate beats. This suggests that intensity accents may result in more clear-cut outcomes. The next two experiments were, therefore, designed to investigate the role of intensity accentuation in rhythmic interpretation.

\section{EXPERIMENT 4 \\ The Effect of Element Intensity on the Rhythmic Interpretation of Polyrhythms of Two Pulse Trains}

The purpose of Experiment 4 was to investigate the effects of intensity accentuation on rhythmic interpretation. Music theorists are unanimous in declaring that louder elements become predominant and thereby determine the rhythmic interpretation (e.g., Cooper \& Meyer, 1960). In the present experiment, there were several variations in accentuation. In some cases, one, two, or all elements of one pulse train were accented; for example, the elements of a 4-pulse train might have been Hhhh (Llli), HhHh (LILl), or HHHH (LLLL), where hs and ls represent high- and low-pitch elements and capitals indicate accented elements. In other cases, elements of both pulse trains were accented. The results demonstrated that intensity accentuation was indeed a prepotent cue. Accented elements determined the choice of the meter rhythm, although individual response patterns with respect to pitch and timing also affected rhythmic interpretation.

\section{Method}

Subjects. There were 25 subjects.

Polyrhythms. One polyrhythm was used: $4 \times 5$. This polyrhythm was chosen because it yielded the widest range of rhythmic interpretations (see Experiment 1 and Handel \& Oshinsky, 1981). The tones of the two pulse trains were separated by a musical fourth, such that in half the instances one pulse was $440 \mathrm{~Hz}$ (musical A4) and the second was $586 \mathrm{~Hz}$ (musical D5), and in half the instances the frequencies of the pulse trains were reversed. The intensity accent was a 15-dB SPL increase in loudness; the softer tones were approximately $55 \mathrm{~dB} \mathrm{SPL}$, and the accented elements were $70 \mathrm{~dB}$ SPL.

Presentation rates. There were five presentation rates: 3.0, 2.4, $1.8,1.2$, and $.6 \mathrm{sec}$.

Intensity accentuation. In total, there were 16 conditions which may be broken into two parts. In the first part, which comprised 12 polyrhythms, some or all of the elements of one pulse train were accented, while all elements of the second pulse train were nonaccented. There were three ways in which the elements of one pulse train were accented: (1) all elements were accented; (2) only the first and third elements were accented (e.g., XxXX for 4-pulse 
trains, $\mathrm{XxXxx}$ for 5-pulse trains; and (3) only the first element was accented. The accented pulse train could have been either low or high pitch and could have been either the 4- or the S-pulse train, thereby generating 12 conditions. In the second part, which comprised four polyrhythms, the elements of both pulse trains were accented. In one instance, all elements of both pulse trains were accented. In the second instance, the first and third elements of both pulse trains were accented. For both, the 4-pulse train could be the low-pitch tone and the 5-pulse train, the high-pitch tone, or vice versa, thereby generating the remaining four conditions.

Experimental conditions. There were a total of 80 conditions resulting from the 16 intensity accent possibilities and the five presentation rates. Each subject was presented all 80 conditions in a 75-min experimental session.

\section{Results}

Although intensity accentuation was the dominant predictor of rhythmic interpretation, there were three strategies.

(1) The first stiategy, illustrated in Figure 10 was the simplest of all. These subjects $(N=8)$ tapped a $4 \times 5$ cross-rhythm at the slower rates. At the faster rates, if only one pulse train was accented, subjects tapped in synchrony with that pulse train (Panels A and B). If both pulse trains were accented (shaded circles and squares), a 5-pulse-train meter was preferred down to the 1.8 -sec/repetition rate, while the 4-pulse-train meter was preferred at the faster rates (Panel C).

(2) The second strategy $(\mathrm{N}=12)$ was determined by intensity accentuation, element frequency, and timing. This strategy is illustrated in Figure 11. In Panel A, the accented 5-pulse-train meter was preferred. If the accented 5-pulse train was the low-pitch element, the percentage of choice was roughly $20 \%$ greater than if it was the high-pitch element. The identical pattern was found when the 4-pulse train was accented (Panel B): a 4-pulse-train meter was the preferred rhythmic interpretation, and the percent choice was $25 \%$ greater if it was the low-pitch tone. If both pulse trains were accented (Panel C), a 5pulse-train meter was preferred at slower rates and a

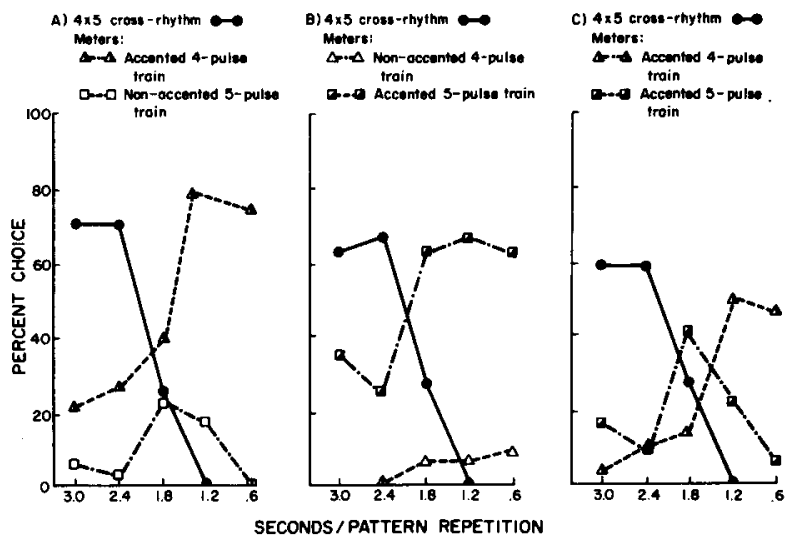

Figure 10. Intensity accentuation: Rhythmic interpretation I based on cross-rhythms and accented pulse-train meter rhythms.

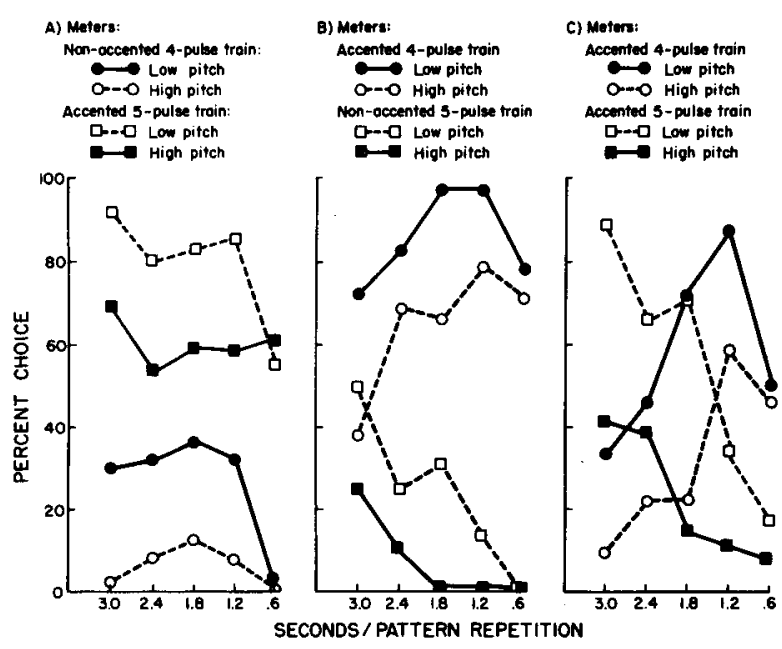

Figure 11. Intensity accentuation: Rhythmic interpretation 2 based on accented and pitch meter rhythms.

4-pulse-train meter was preferred at the faster rates. Here, too, a low-pitch meter was preferred to a highpitch meter.

(3) The third strategy was the most unique and involved the smallest number of subjects $(\mathrm{N}=4)$. Here subjects tapped in synchrony to all elements of the accented pulse train if one or all of the elements were accented and none of the elements of the second pulse train were accented (e.g., Hhhh $\times$ lllll, HHHHH $\times$ llll, hhhhh $\times$ Lllll, hhhh $\times$ LLLLL). However, if the first and third elements of one pulse train were accented or if elements of both pulse trains were accented, then subjects tended to tap a unit rhythm, on the simultaneous occurrence of the elements of both pulse trains (48\%).

In addition to serving as a dominant cue for rhythmic organization, intensity accents also served a more general temporal function: they allowed all subjects to synchronize with the elements of a pulse train at faster rates. Obviously, this statement holds only for those polyrhythms in which one or two of the elements are accented. If all elements of a pulse train are accented, then accents cannot serve as "markers." At the fastest rate, for those polyrhythms in which all elements of each pulse train were either accented or nonaccented so that the accented elements would not serve as markers, the percentage of responses that were nonrhythmical according to the previously detailed criteria was $60 \%$. In contrast, if only one or two elements of a pulse train were accented, the percentage of nonrhythmical responses was $8 \%$. It appeared that the pattern of accented and nonaccented elements allowed successful synchronization. Moreover, the pattern need not be perfectly regular, since the irregular accent pattern HhHhh or LlLll also led to successful synchronization. 


\section{Discussion}

Intensity accentuation was an important determinant of perceived rhythm. The accents could determine the meter rhythm irrespective of other factors, or the accents could determine the meter rhythm in conjunction with element pitch and timing. This meter could be achieved by tapping every element or by tapping only the accented elements of that pulse train.

It should be noted that the interpretation strategies found for nonaccented $4 \times 5$ polyrhythms in Experiment 1 and those found in the present experiment are essentially the same. In Experiment 1, one subject cluster tapped a cross-rhythm at slow rates and then tapped a low-pitch meter or the slower 4-pulse-train meter at the faster rates. A similar pattern was found here-cross-rhythms at slower rates and meter rhythms at faster rates. The accents, however, led to meter rhythm based on the accented pulse train without regard to pitch or rate. In Experiment 1, the second subject cluster tapped faster meters at slow rates and slower meters at fast rates. The second cluster of subjects found here used a similar strategy when both pulse trains were accented: faster meters at slower rates, slower pulse-train meters at faster rates. When only one pulse train was accented, though, subjects did not switch meter: the accented pulse train was chosen as the meter at all presentation rates. The intensity accents overcame temporal and pitch preferences underlying meter selection. The general, but weak, preference for a meter based on the low-pitch element acted only to modify the predominant preference for the accented pulse-train meter.

The same hesitations mentioned previously concerning the generality of results arising from the rather simple polyrhythms composed of two pulse trains must be raised again. For this reason, the fifth and final experiment investigated the effect of intensity accentuation on the rhythmic interpretation of more complex polyrhythms composed of three pulse trains.

\section{EXPERIMENT 5 \\ The Effect of Element Intensity on the Rhythmic Interpretation of Polyrhythms Composed of Three Pulse Trains}

The purpose of Experiment 5 was to investigate the effect of intensity accentuation on the rhythmic interpretation of more complex polyrhythms composed of three pulse trains. These polyrhythms allow for diverse relationships among pulse trains, frequency, and accentuation which cannot occur for polyrhythms composed of two pulse trains.

The $3 \times 4 \times 5$ polyrhythm was selected, on the basis of the results of Experiment 2, because it displayed a wider range of rhythmic interpretations than other polyrhythms. In that experiment, two clusters of sub- jects displaying quite different rhythmic strategies were found: one cluster of subjects displayed a strong preference for low-pitch meters based on pitch, and a second cluster of subjects displayed a preference for cross-rhythms generated by two or all three of the pulse trains. Thus, it is possible to determine if intensity accentuation acts to overcome pitch preferences, acts to preclude cross-rhythms generated by two or three pulse trains, and/or acts to overcome meter preference based on timing between elements of one pulse train.

\section{Method}

Subjects. There were 20 subjects.

Polyrhythms. The single polyrhythm used was $3 \times 4 \times 5$. In all polyrhythms, either two pulse trains were the same frequency and one differed or all three pulse trains were the same frequency. The tones of the pulse trains were $252 \mathrm{~Hz}(\mathrm{C4}), 330 \mathrm{~Hz}$ (E4), and $392 \mathrm{~Hz}$ (G4). If elements of all three pulse trains were identical, the low tone $(252 \mathrm{~Hz})$ was used. The nonaccented elements were $55 \mathrm{~dB}$ SPL, and the accented elements were $70 \mathrm{~dB}$ SPL, exactly as in the previous experiment.

There were a total of 22 polyrhythms. In 4, all three pulse trains were of the same frequency: in one case, all three pulse trains were nonaccented, and in three cases, all elements of one pulse train were accented. In 18, two pulse trains had the same frequency and one pulse train contrasted. In 6 of these polyrhythms, all elements were unaccented: the contrasting tone could be either high or low pitch and could be one of three pulse trains, thereby generating six total possibilities. In 12 polyrhythms, all elements of one of the two identical-frequency pulse trains were accented; there were two possibilities, since either pulse train could be accented. For example, the 3- and 4-pulse trains might be high pitch and the 5-pulse train low pitch (e.g., $h \times h \times l$ ). Then, the 3-pulse train could be accented $(\mathrm{H} \times \mathrm{h} \times \mathrm{l})$ or the 4-pulse train could be accented $(\mathrm{h} \times \mathrm{H}$ $x$ I). The 12 cases arise from the six ways of constructing polyrhythms composed of one contrasting-frequency pulse train combined with two ways of accenting each such polyrhythm.

Pattern repetition rates. Four rates of presentation were used: $2.4,2.0,1.6$, and $1.0 \mathrm{sec}$.

Experimental design. There were 88 conditions: 22 frequencyaccent possibilities $\times 4$ presentation rates. The 20 subjects were presented all 88 conditions in a single 90 -min session.

\section{Results}

Preliminary analyses indicated that, on the whole, all subjects displayed a similar response pattern. On this basis, all results presented below were based on 20 subjects.

The polyrhythms composed of nonaccented pulse trains of the identical frequency served as a reference to investigate the effect of intensity accentuation and pitch differences. For this polyrhythm, there was no predominant response, although there was a tendency to tap a $3 \times 4 \times 5$ cross-rhythm at the slowest rates $(38 \%)$ and tap once per repetition at the two fastest rates $(40 \%)$. For polyrhythms in which elements of one pulse train were accented but there were no pitch differences, a meter rhythm based on the accented pulse train was almost the unanimous choice. The percentages of accent meter rhythms were $94 \%$, $95 \%$, and $94 \%$ for accented 3-, 4-, and 5-pulse trains, respectively. 
For polyrhythms in which the pitch of the elements of one pulse train contrasted but there was no intensity accentuation, rhythmic interpretation was determined by a meter preference for the contrasting pulse train, by a meter preference for the low-pitch pulse train, and by a meter preference for the slower 3- or 4-pulse train, particularly at the faster presentation rates. On this basis, if the contrasting pitch was the low tone (i.e., the two identical tones were high pitch), then the meter rhythm based on that pulse train was chosen $95 \%, 88 \%$ and $74 \%$ of the time for the 3-, 4-, and 5-pulse trains, respectively. If the contrasting pitch was the high tone, then the percentage choice of a high-pitch pulse-train meter was lower: $55 \%, 51 \%$, and $29 \%$ for the 3-, 4-, and 5-pulse trains, respectively. In sum, making one pulse train distinctive in either intensity or pitch led to a meter based on that pulse train.

If both intensity and pitch differences occurred, the basis for rhythmic interpretation changed. Here, polyrhythms composed of two low-pitch pulse trains yielded rhythms different from those of polyrhythms composed of two high-pitch pulse trains. For polyrhythms composed of two low-pitch tones and one high-pitch tone, the rhythmic interpretation was a meter based on the accented low-pitch pulse train. The percentage choice for the accented low-pitch 5pulse train ranged from $83 \%$ at the 2.4-sec/repetition rate to $55 \%$ at the $1.0-\mathrm{sec} /$ repetition rate. The percentage choice for the accented low-pitch 4-pulse train ranged from $93 \%$ to $68 \%$, and the percentage choice for the accented low-pitch 3-pulse train ranged from $82 \%$ to $88 \%$. These results demonstrated that, in addition to the preference for the accented pulse train, the general temporal consideration of basing the meter on the faster pulse train at slower rates and on the slower pulse train at faster rates also affected the response. The rhythmic interpretation based on accentuation, frequency, and timing found here should be contrasted to the preferred meter interpretation based on the contrasting high-pitch tone when all pulse trains were unaccented (above and in Experiment 2). The intensity accent thus dominated pitch differences in rhythmic interpretation.

A different pattern of outcomes occurred when two pulse trains were high pitch and one pulse train was low pitch. Here, the determining meter-choice factor was the pulse-train assignment of the contrasting low-pitch tone, and not the assignment of the accented high-pitch tone. The rhythmic interpretations are portrayed in Figure 12. In the first instance (Panel A), the 3-pulse train was the low pitch, as found in the two polyrhythms $\mathrm{l} \times \mathrm{H} \times \mathrm{h}$ and $\mathrm{l} \times \mathrm{h} \times \mathrm{H}$. The percentage choice of the 3-pulse-train meter was averaged across the two polyrhythms, and the percentage choice of the accented high-pitch pulse train (i.e., the 4-pulse train in the $\mathrm{I} \times \mathrm{H} \times \mathrm{h}$ polyrhythm and the 5-pulse train in the $1 \times \mathrm{h} \times \mathrm{H}$ polyrhythm) was

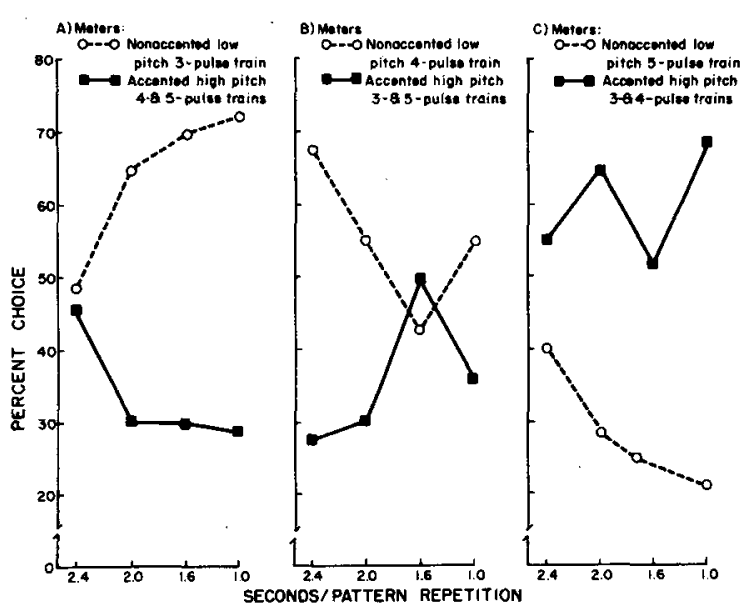

Figure 12. The effect of pitch contrast, intensity accentuation, and pulse-train rate on meter choice.

averaged. The nonaccented high-pitch pulse train was rarely chosen. The same procedure was followed for the other possibilities.

If the contrasting low-pitch element was the 3pulse train, it was preferred as the meter to the accented high-pitch 4- or 5-pulse train (Panel A). If, however, the low-pitch element was the 5-pulse train (Panel C), the accented high-pitch 3- or 4-pulse train was preferred at all presentation rates. The instances in which the low-pitch element was the 4-pulse train represent an intermediate case (Panel B): the lowpitch 4-pulse train was highly preferred at the slower rates, whereas there were roughly equal preferences for the low-pitch 4-pulse train and the accented high-pitch 3- or 5-pulse trains at the faster rates.

\section{Discussion}

These results illustrated once again that rhythmic interpretation is a function of many interrelated cues. Here, the perceived rhythm was determined by intensity accentuation, pitch assignment of the pulse trains, intensity accentuation overlaid on the pitch assignments, and the timing of pulse trains. The various cues are substitutable. Meter preferences can be brought about by intensity accentuation in the absence of pitch difference, by pitch difference in the absence of intensity accentuation, and by the interaction of intensity accentuation and pitch difference, that is, the effect of intensity accentuation depended on the specific pitch contrast. This not to say that rhythm is completely labile, because there are temporal constraints which cut across intensity and pitch manipulations.

In comparison with results obtained for the nonaccented $3 \times 4 \times 5$ polyrhythm in Experiment 2, the overall effect of accentuation was to reduce sharply the use of cross-rhythms. The intensity accentuation acted to make identical-frequency pulse 
trains dissimilar and noncoordinate, thereby precluding cross-rhythms combining those pulse trains. The rhythm shifted to a meter, based on a single pulse train. The preference for a meter rhythm was multiply determined, being contingent on the relationships among element pitch, element timing, element accentuation, and pitch contrast; no single factor could explain meter choice.

\section{GENERAL DISCUSSION}

One common theme running through the five experiments reported here is the contextual nature of rhythmic interpretation. Previous work by Handel and Oshinsky (1981) had demonstrated that rhythmic interpretation was contextual with respect to tempo; the rhythm differed as a function of rate. The present work generalized this conclusion into a number of new domains. The perceived rhythm is contextual with respect to polyrhythm configuration, and with respect to element frequency, element intensity, and element duration. Each of these factors influences the perceived rhythm, but the effect depends on the values of all other factors. For example, the effect of element duration depends on element frequency, presentation rate, and the polyrhythm configuration. In addition to the interactive, contextual nature of the stimulus variables, there are the striking individual differences in the way that each "complex" of factors is utilized in the rhythmic interpretation.

The rhythm does not exist at any specific level in a polyrhythm (Yeston, 1976). It emerges as an impression, coming out of the interplay among the levels. The rhythmic levels can be of the same sort, as are the pulse trains of a polyrhythm, or may be of quite different types, as found in other styles of composition. Each rhythmic level, however, functions as both a figure and a ground; it becomes part of the perceived rhythm, yet simultaneously is part of the supporting framework for other rhythmic levels. This makes theorizing about rhythm extremely difficult. There are no basic units out of which rhythm can be built. Although the goal of analysis is still possible, prediction may be impossible in novel contexts due to the contextual, interacting nature of rhythmic levels in generating the rhythm perceived.

Nonetheless, we can describe two general factors that underlie rhythmic interpretation: (1) timing and (2) polyrhythm configuration.

(1) The first factor that underlies rhythmic interpretation is the timing and tempo of the polyrhythm. Across all experiments, there are consistent changes in rhythmic interpretation as a function of presentation rate. At slower presentation rates, cross-rhythms or faster meters were preferred, whereas at faster presentation rates, slower meters or even unit meters were preferred. These preferences seem to be attrib- utable to both perceptual and motoric considerations.

Perceptually, a rhythm can serve different roles. In one role, a rhythm can provide a lattice, framework, or clock against which the foreground can be structured. The ability of a pulse train to serve as a frame is limited by an interelement timing window between 200 and $800 \mathrm{msec}$, within which individual elements can provide a beat. At longer intervals, the elements appear unconnected and disjointed; at shorter intervals, the elements appear to be subjectively grouped. This timing window may seem unduly restricted, particularly at the longer end. Previous work, summarized by Fraisse (1978), suggests that two tones separated by intervals as long as $1.5 \mathrm{sec}$ can be integtrated; moreover, there are many compositions performed at slower tempos (i.e., an 800 -msec interelement interval would be equivalent to a $75-\mathrm{MM}$ tempo). Clearly, exact limits for temporal integration are not fixed rigidly and depend on the specific context. For example, in the present research there are polyrhythms in which a pulse train can serve as the meter at longer interelement intervals. One preferred rhythm for the $2 \times 5 \times 7$ polyrhythm was a 2-pulse-train meter, and this meter was preferred for interelement intervals as long as $1.5 \mathrm{sec}$. In spite of these exceptions, we believe that these timing constraints for meters are applicable generally. In musical instances with a slow metronome tempo, the next smaller denominator (e.g., eighth notes if the tempo specifies quater notes) may be the true timing element (see Read, 1969), and the timing of those elements would satisfy the interelement interval window.

In another role, a rhythm can be a foreground, that is, a cross-rhythm composed of two or even three pulse trains. For cross-rhythms, there are two kinds of interelement intervals: the constant intervals between elements of one pulse train and the varying intervals between elements of different pulse trains. These variations in timing would seem to make predictions solely on the basis of interelement intervals problematic. The preference for cross-rhythms was, in fact, more variable with respect to presentation rates and suggests that other esthetic factors may influence rhythm choice. Some cross-rhythms are more complex phenomenally - the timing between elements is more syncopated-and this may determine preference and choice.

Motorically, the rhythm provides the stimulus for "body" synchronization. The tempo obviously will affect the nature of the synchronization. At slow tempos, subjects are able to tap both cross-rhythms and faster meter rhythms. It may be more difficult to tap in synchrony with the more complex crossrhythms, and indeed they are preferred less frequently. At faster tempos, the ability to synchronize 
the motor tapping is of greater importance. There does seem to be an upper limit on synchronization to a repeating isochronous auditory signal of about 6 or 7 elements/sec (Bartlett \& Bartlett, 1959). The upper limit of synchronization to the variable cross-rhythms should be lower.

At this point, it is impossible to partition the manner in which perceptual and motoric constraints bring about changes in rhythmic interpretation due to tempo. Historically, rhythmic apprehension and muscle movements were thought to be intricately linked: musical structures must initiate muscle movements in order for the impression of rhythm to emerge. Although movements usually accompany rhythmic experience, they certainly are not the cause of it. However, the connection between body movement and rhythm experience is convincing regardless of any causal link. This connection, taken along with the required synchronous tapping response, suggests that the rhythmic interpretation must be a function of both perceptual and motoric factors.

It is important to question whether the synchronous tapping response utilizing one limb limits the expression of rhythmic experience. People may tend to "distribute" different rhythmic lines to different body members. One hand may tap each note, the second hand may tap each beat, and a leg may tap each measure. Alternative response modes may, indeed, lead to "richer" rhythmic interpretations. Moreover, Shaffer (1981) has demonstrated that skilled pianists can independently vary the timing of pulse trains played with each hand. These variations lead to expressive changes that must surely affect the rhythmic interpretation. We do not know whether there are limits to the possibilities for musical performance, either in terms of the performer's ability to produce the desired timings or in terms of the listener's ability to perceive the initial rhythmic structure and the improvisations off this structure.

(2) The second factor that underlies rhythmic interpretation is the polyrhythm configuration. These experiments have probed two aspects of configuration-the relationships among the pulse-train rates and the relationships among the pulse-train pitchesand both consistently affected the rhythmic interpretation.

We can point to two effects of the timing relationships among pulse trains. First, if the two or three pulse trains have nearly identical rates (e.g., $2 \times 3$, $3 \times 4,4 \times 5,3 \times 4 \times 5$ ), subjects tended to select the low-pitch pulse train as the polyrhythm meter. For these polyrhythms, more than one pulse train will satisfy the timing constraints at each presentation rate, and this might induce subjects to utilize the alternate pitch attribute. However, pitch meters did not occur for other polyrhythms composed of more widely spaced pulse trains at presentation rates in which two or more pulse trains satisfied the timing constraints. Pitch meters therefore seem restricted to polyrhythms in which the pulse trains have similar rates. Second, the distribution of pulse-train rates influences the preference for cross-rhythms and alternative types of meter rhythms. This effect of configuration was discussed in Experiment 2 . In that discussion, the $2 \times 3$ polyrhythm was compared with the $2 \times 3 \times 7$ polyrhythm and the $2 \times 5$ polyrhythm was compared with the $2 \times 5 \times 7$ polyrhythm to isolate the effect of the 7-pulse train. To summarize, for the $2 \times 3 \times 7$ polyrhythm, the 7 -pulse train emphasized the rate similarity between the 2 - and 3-pulse trains, leading to an increased use of $2 \times 3$ cross-rhythms but a decreased use of both 2-pulse- and 3-pulse-train meters as compared with $2 \times 3$ polyrhythms. In contrast, for the $2 \times 5 \times 7$ polyrhythm, the 7-pulse train emphasized the rate difference between the 2 - and 5pulse trains, leading to a decreased use of $2 \times 5$ crossrhythms but an increased use of 2-pulse-train meters as compared with the $2 \times 5$ polyrhythm. It should be noted that for both the $2 \times 3 \times 7$ and the $2 \times 5 \times 7$ polyrhythms the use of the "middle"-pulse-train meter was minimal. Therefore, the pulse-train configuration can act to highlight similarities and differences among pulse-train rates and thereby change the rhythmic interpretation.

We can point to several effects of the pitch relationships among pulse trains. These emerged for polyrhythms composed of three pulse trains when two pulse trains were the identical pitch and one pulse train differed. For interpretation strategies in which cross-rhythms predominated $(2 \times 3 \times 7$ polyrhythm, Figure 3; 3×4 55 polyrhythm, Figure 7), use of cross-rhythms increased if the contrastingpitch pulse train was not part of the cross-rhythm (see Figure 3, Panel B), but the use of the crossrhythm decreased if the contrasting-pitch pulse train was one of the cross-rhythm pulse trains (see Figure 3, Panels $C$ and D). The results for the $3 \times 4 \times 5$ polyrhythm differed: The two identical-pitch pulse trains formed the preferred cross-rhythm at slow presentation rates, and the contrasting-pitch pulse train became the preferred meter at the fast presentation rates. For interpretation strategies in which meter rhythms predominated $(2 \times 3 \times 7$ polyrhythm, Figure 2; $2 \times 5 \times 7$ polyrhythm, Figures 4 and $5 ; 3 \times 4 \times$ 5 polyrhythm, Figure 6), the contrasting-pitch pulse train gained in preference. Thus, the effect of pitch contrast depended on the polyrhythm configuration and on the interpretation strategy. In addition, the effect of intensity accentuation depended on the nature of the pitch contrast. As shown in Experiment 5 , an intensity accentuation overlaid on one of two low-pitch pulse trains affected rhythm interpretation differently from the way in which intensity accentuation overlaid on one of two high-pitch pulse trains did.

These outcomes suggest a view of rhythmic interpretation which may force us to modify our goals for a theory of rhythm. No current theory seems ade- 
quate to portray or encompass rhythmic diversity. Moreover, it is unclear whether there is a level or levels at which generalizations about rhythm can emerge.

Although it is true that if we define perception as the determination of which syllables or notes carry the stress and which do not, then a simpler theory of rhythm based on hierarchical grouping principles may suffice both in speech (e.g., Martin, 1972) and in music (Cooper \& Meyer, 1960). On the other hand, if we define perception as to what a given stress pattern signifies, then it seems almost a truism that perceiving is impossible without taking the entire interaction into account, both in speech (Chomsky \& Halle, 1968) and in music (Yeston, 1976). The perception of the overall musical or linguistic configuration cannot happen without some integration of these layers. What is needed are theories which predict a range of alternative rhythmic interpretations coming from the interaction of rhythmic levels.

\section{REFERENCES}

BAntlett, N. R., \& BAntletT, S. C. Synchronization of a motor response with an anticipated sensory event. Psychological Review, $1959,66,203-218$.
Chomsky, N., \& HALLE, M. The sound pattern of English. New York: Harper \& Row, 1968.

COOPER, G., \& MEYER, L. B. The rhythmic structure of music. Chicago: University of Chicago Press, 1960.

Erickson, R. Sound structure in music. Berkeley: University of California Press, 1975.

Fraisse, P. Time and rhythm perception. In E. C. Carterette \& M. P. Friedman (Eds.), Handbook of perception (Vol. 8). New York: Academic Press, 1978.

HANDEL, S., \& Oshinsky, J. S. The meter of syncopated auditory polyrhythms. Perception \& Psychophysics, 1981, 30, 1-9.

Jones, A. M. Studies in African music. London: Oxford University Press, 1959.

Martin, J. G. Rhythmic (hierarchical) versus serial structure in speech and other behavior. Psychological Review, 1972, 79, 124-145.

OshinskY, J. S., \& Handel, S. Syncopated auditory polyrhythms: Discontinuous reversals in meter interpretation. Journal of the Acoustical Society of America, 1978, 63, 936-939.

Radocy, R. E., \& Boyle, J. D. Psychological foundations of musical behavior. Springfield, Ill: Thomas, 1979.

READ, G. Music notation. Boston: Crescendo, 1969.

Shaffer, L. H. Performances of Chopin, Bach, and Bartok: Studies in motor programming. Cognitive Psychology, 1981, 13, 325-376.

Yeston, M. The stratification of musical rhythm. New Haven: Yale University Press, 1976.

Zuckerkand, V. The sense of music. Princeton, N.J: Princeton University Press, 1959.

(Manuscript received July 26, 1982; revision accepted for publication April 4, 1983.) 